\title{
Prediction of total and visceral fat contents using anthropometric measures of adiposity in women
}

\author{
T P Weerarathna ${ }^{1}$, S Lekamwasam ${ }^{2}$ and M Rodrigo $^{3}$
}

\begin{abstract}
Background Although techniques such as dual energy xray absorptiometry (DXA) and quantitative CT are available to estimate global and regional adiposity, anthropometric measurements are often used to detect adiposity in clinical practice.

Objectives To assess the association between the anthropometric measurements of obesity with total and regional fat mass determined by DXA.

Design A cross-sectional, descriptive study.

Patients and method 106 healthy women volunteers, aged between 30 and 54 years were studied. Anthropometric measurements including body mass index (BMI), waist circumference (WC), hip circumference (HC), height adjusted WC (WC/HT), waist-hip ratio (WHR), and skin-fold thickness (SFT) over triceps, infrascapular, and iliac regions were made. All women underwent assessment of total fat mass (TFM) and visceral fat mass (VFM) using a Hologic DXA scan.

Results TFM and VFM showed positive correlations with all the anthropometric measurements examined, the strongest correlation was with BMI ( $r=0.89$ and 0.77 for TFM and VFM respectively, $p<0.001$ ). Correlations of TFM with WC, $\mathrm{HC}$, and $\mathrm{WC} / \mathrm{HT}$ were $0.72,0.87$, and 0.65 , $(p<0001$ for all) respectively. Corresponding figures for VFM were $0.73,0.74$, and 0.68 , ( $p<0001$ for all). WHR showed a poor correlation with TFM $(r=18, p=0.09)$ and VFM $(r=0.33, p=0.002)$. SFTs measured at three sites showed less strong correlations with TFM and VFM ( $r=$ 0.48 to $0.69, p<0.001)$.

Conclusions BMI has the strongest association with total and visceral fat mass among these women. Waist and hip circumferences showed high correlations with total and visceral fat mass, but adjusting waist circumference for height did not improve the correlation.
\end{abstract}

\section{Introduction}

Obesity is regarded as a relative measure of fatness or adiposity of an individual. Body mass index (BMI) is the most widely used anthropometric measurement to estimate adiposity in clinical practice. BMI is a measure of an individual's weight in relation to height, and is considered a clinical surrogate of the total fat mass.

With a large body of research showing a link between accumulation of fat in the abdomen and adverse metabolic complications, there has been a recent interest in the surrogate measurements of abdominal or visceral adiposity [1]. Visceral fat, the fat accumulated in the abdominal region, is metabolically more active than fatty tissue elsewhere [2]. Visceral fat and associated humoral mediators such as leptin and adiponectin are thought to contribute to insulin resistance and many metabolic and cardiovascular diseases [3]. Estimation of visceral fat with sensitive anthropometric measurements would help in the early identification of individuals at risk of these diseases.

WC and WHR are the two widely used anthropometric indices of visceral adiposity. Measurement of SFT, which is an estimate of the subcutaneous fat content has been used to asses adiposity in younger age groups [4].

Apart from the anthropometric measurements, several non-invasive investigations are available to measure the fat mass [5]. DXA and quantitative CT measure the total fat mass and its distribution in different regions of the human body [6]. DXA gives more precise information on the total fat mass and the visceral fat content than anthropometric measurements [7].

Although BMI and WC are widely used in clinical settings, their limitations are well known. The issue of selecting a universal measurement to detect adiposity becomes more complex as the percentage and the distribution of adipose tissue in human bodies have a wide variation according to age, sex, and the ethnicity of the individual [8].

Screening individuals for early detection of noncommunicable diseases has become a matter of public health importance. Studies are needed in Asian populations to ascertain the most valid and appropriate anthropometric measurements in the detection of different types of obesity. Owing to the limited availability of studies examining these associations in Asian populations, there is uncertainty regarding the cut-off values of BMI and WC applicable to Asian populations.

${ }^{1}$ Centre for Lipid Disorders, ${ }^{2}$ Centre for Metabolic Bone Diseases, and ${ }^{3}$ Department of Anatomy, Faculty of Medicine, Galle, Sri Lanka.

Correspondence: TPW, e-mail: <thilakpw@yahoo.com>. Received 15 June and revised version accepted 4 October 2008. Conflicts of interest: none declared. 
In the present study, we intended to evaluate the association of commonly used anthropometric measurements of obesity namely, BMI, WC, height adjusted WC (WC/HT), hip circumference (HC), WHR and SFT with total and visceral fat masses measured by DXA, and to determine how one may predict body fat content, both total and visceral, using each of these measurements.

\section{Materials and methods}

A group of previously healthy, premenopausal women volunteers aged 30 years or more, was included in the study. Approval for the study was obtained from the ethics review committee of the Faculty of Medicine, Galle. Individuals were recruited by open invitations displayed at healthcare institutions in southern Sri Lanka. After obtaining written consent, all participants were interviewed with regard to their health and medicinal drug use. Those with diabetes, hypertension, epilepsy, chronic renal or liver disease, hyperlipidaemia, ischaemic heart disease, endocrine disease, prolonged inflammatory disease, and those who were on medications such as corticosteroids, hormone replacement, antiresorptive drugs, oral hypoglycaemic agents, thyroxine and lipid lowering therapy were excluded.

The following anthropometric measurements were taken by one investigator. Weight was measured while wearing light clothes and after emptying the urinary bladder. Height was measured without shoes using stadiometer. After removing undergarments, WC was measured with a measuring tape at the midpoint between the iliac crest and the lower border of the rib cage. HC was taken at the widest diameter across the hips while wearing light clothes. SFT was measured with a skin caliper at three places - the midway of upper arm over the triceps, over the anterior-superior iliac crest, and just medial to the scapular border. The average of two SFT measurements at each site was considered as the final measurement.

All individuals underwent DXA scan using a Hologic Discovery scanner (Hologic Inc, Bedford, MA, USA). TFM and VFM were estimated using the standard analytical software provided by the manufacturer. One technician performed all DXA scans and analysed them in one session to minimise operator variability.

\section{Statistics}

113 individuals were recruited to the study. Seven DXA images were unsuitable for analysis and data of 106 women were included in the final analysis. Characteristics of the 106 women included in the final analysis are given as mean and standard deviations (table 1). Waist circumference was adjusted for height by dividing the WC from the height of subjects (WC/HT). All anthropometric measurements and fat estimations were normally distributed. Pearson correlation was used to asses the association between the anthropometric measurements (BMI, WC, WHR, SFT and WC/HT) with the TFMs and VFMs.

Table 1. Characteristics of 106 women included in the analysis

\begin{tabular}{lrl}
\hline Measurement & Mean & SD \\
\hline Age (years) & 42.10 & 6.00 \\
Weight $(\mathrm{kg})$ & 57.54 & 8.88 \\
Height $(\mathrm{m})$ & 1.54 & 0.05 \\
*BMI $\left(\mathrm{kg} / \mathrm{m}^{2}\right)$ & 24.32 & 3.55 \\
Waist circumference $(\mathrm{cm})$ & 84.68 & 8.97 \\
Hip circumference $(\mathrm{cm})$ & 96.67 & 7.46 \\
**WHR & 0.87 & 0.05 \\
***SFT over triceps $(\mathrm{mm})$ & 19.48 & 5.65 \\
$* * *$ SFT over iliac crest $(\mathrm{mm})$ & 28.81 & 7.03 \\
$* * *$ SFT over scapula $(\mathrm{mm})$ & 27.25 & 6.04 \\
Height adjusted waist circumference $(\mathrm{cm})$ & 55.08 & 6.13 \\
Total fat mass $(\mathrm{kg})$ & 20.29 & 5.09 \\
Visceral fat mass $(\mathrm{kg})$ & 7.65 & 2.09 \\
\hline
\end{tabular}

*BMI= Body mass index, ${ }^{* *}$ WHR= Waist-hip ratio, ${ }^{* * * S F T=S k i n-f o l d ~ t h i c k n e s s ~}$ 
Regression analysis was performed between fat mass (dependent variable) and each anthropometric measurement (independent variable). Regression model was fitted to examine the strongest predictor of the fat mass by including all anthropometric measurements as independent variables in the model and then excluding the weak associations in step-wise fashion. Two-tailed $\mathrm{p}<0.05$ was taken as the level of statistical significance and SPSS version 10 for Windows was used for analyses.

\section{Results}

The age of women included in the study ranged from 30 to 54 years with a mean (SD) of 42.0 (6.0) years. $6(5.6 \%)$ women had BMI above $30 \mathrm{~kg} / \mathrm{m}^{2}$, and 8 (7.5\%) women had BMI below 20. The TFM showed positive and significant correlations with all anthropometric measurements except the WHR. All anthropometric measurements were positively and significantly correlated with VFM. BMI showed the highest correlation with both TFM and VFM, and correlations with HC and WC were marginally less. Adjusting the WC for height did not improve the association. WHR showed no significant correlation with TFM and correlation with VFM was moderate. The commonly used anthropometric indices of visceral adiposity such as WC, WHR and WC/HT had no superior ability to predict visceral fat mass than BMI. Skinfold thickness at all three sites showed significant association with TFM and VFM but the degree of association was not as strong as those seen with BMI, WC or HC. Although 75-78\% of TFM could be predicted using either BMI or HC, only 54-59\% of VFM could be predicted using the skin-fold thickness (tables 2 and 3). When the regression model was fitted to exclude weak associations stepwise, BMI remained to be the strongest predictor of both TFM and VFM.

Table 2. Association between the total fat mass and the anthropometric measurements

\begin{tabular}{|c|c|c|c|c|}
\hline & Correlation (r ) & $r^{2}$ & $\begin{array}{l}\text { Regression coefficient } \\
\text { (95\% confidence intervals) }\end{array}$ & $P^{*}$ \\
\hline Body mass index & 0.89 & 0.78 & 1210.7 (1082.2 to 1339.2$)$ & $<0.001$ \\
\hline Waist circumference & 0.72 & 0.52 & 387.8 (311.0 to 464.5$)$ & $<0.001$ \\
\hline Hip circumference & 0.87 & 0.76 & $564.4(499.3$ to 629.4$)$ & $<0.001$ \\
\hline **WC/HT & 0.64 & 0.41 & 505.2 (381.2 to 629.3$)$ & $<0.001$ \\
\hline Waist-hip ratio & 0.18 & 0.03 & $13169.0(-3715.8$ to 30053.9$)$ & 0.090 \\
\hline ***SFT: triceps & 0.66 & 0.43 & 560.3 (427.0 to 693.5) & $<0.001$ \\
\hline SFT: scapular & 0.65 & 0.43 & $526.6(402.2$ to 650.9$)$ & $<0.001$ \\
\hline SFT: iliac & 0.58 & 0.33 & $399.9(284.7$ to 515.2$)$ & $<0.001$ \\
\hline
\end{tabular}

* p for the regression model fitted with total fat mass as the dependent variable and each anthropometric measurement as the independent variable

**WC/HT: Height adjusted waist circumference $\quad * * *$ SFT: Skin-fold thickness

Table 3. Association between the visceral fat mass and anthropometric measurements

\begin{tabular}{|c|c|c|c|c|}
\hline & Correlation (r) & $r^{2}$ & $\begin{array}{l}\text { Regression coefficient } \\
\text { (95\% confidence intervals) }\end{array}$ & $P^{*}$ \\
\hline Body mass index & 0.77 & 0.59 & $439.9(365.5$ to 514.4$)$ & $<0.001$ \\
\hline Waist circumference & 0.72 & 0.52 & 165.2 (132.8 to 197.6$)$ & $<0.001$ \\
\hline Hip circumference & 0.74 & 0.55 & 203.6 (165.8 to 241.5$)$ & $<0.001$ \\
\hline **WC/HT & 0.67 & 0.45 & $224.2(173.4$ to 275.0$)$ & $<0.001$ \\
\hline Waist-hip ratio & 0.31 & 0.09 & 10775.3(3868.5 to 17682.1 ) & 0.002 \\
\hline ***SFT: triceps & 0.47 & 0.22 & $170.9(105.1$ to 236.8$)$ & $<0.001$ \\
\hline SFT: scapular & 0.63 & 0.40 & 213.2 (159.8 to 266.6$)$ & $<0.001$ \\
\hline SFT: iliac & 0.49 & 0.24 & 142.6(91.1 to 194.1$)$ & $<0.001$ \\
\hline
\end{tabular}

* $\mathrm{p}$ for the regression model fitted with visceral fat mass as the dependent variable and each anthropometric measurement as the independent variable

**WC/HT: Height adjusted waist circumference $\quad * * *$ SFT: Skin-fold thickness 


\section{Discussion}

Obesity and its metabolic and cardiovascular complications are important health issues [9]. Visceral obesity is strongly associated with type 2 diabetes and premature cardiovascular disease [10]. Measures to detect individuals with different types of obesity and recommending appropriate interventions are important. Screening for obesity requires sensitive measurements to predict both the TFM and VFM in the body. Applicability of a measurement is more if it is easily available and convenient to use in clinical settings.

There is no uniform measurement to estimate global or regional fat content in different populations. Because of the ethnic variations in body composition, opinions vary with regard to the best predictor of the fat content in the human body [11]. People of Afro-Caribbean descent have increased skeletal muscle mass and bone mineral content, whereas Asians have lower skeletal muscle mass, low bone mineral content and excess body fat for a given BMI [12].

Results of our study showed BMI to be the best predictor of TFM and VFM. Although other measures of anthropometry were positively associated, they had less strong associations. According to our results, BMI explained the variations of fat mass, both total and regional, better than other variables. BMI alone explained 78\% variation of TFM and 59\% variation of VFM.

Several studies have shown that WC is strongly correlated with BMI, total abdominal fat, subcutaneous abdominal fat and truncal fat [13]. Association of WC with cardiovascular diseases is well recognised. In a major diabetes prevention study WC was the best predictor of development of diabetes among individuals with impaired glucose tolerance [14]. In the WHO MONICA study, the degree of overweight was reflected by the WC and not by the WHR [15]. The currently used definitions of the metabolic syndrome by the International Diabetes Federation and the Adult Treatment Panel III have included WC in preference to BMI [16].

Although it is commonly believed that WC is a surrogate measure of visceral fat, it has a substantial contribution from metabolically different fat contained in the subcutaneous tissue [17]. Several investigators have found that the correlation of WC to visceral fat depends on sex and ethnicity, as it was weaker among women and blacks [18]. The reasons for the different findings of our study could be due to either ethnic or gender differences, or small sample size.

Although WC was not the best predictor of body fat content of these women, it was superior to WHR. Better sensitivity and utility of WC over WHR as an anthropometric measurement of obesity among Asian populations has previously been recognised and was postulated to be due to wider hip circumference in Asian women [19].
A Japanese study of 6141 men and 2137 women investigating the association between BMI, WC, WC/HT and a morbidity index comprising cardiovascular risk factors such as elevated plasma glucose, abnormal lipid profile, and systolic and diastolic blood pressures, reported the highest correlation coefficient between WC/ $\mathrm{HT}$ and the morbidity index for coronary risk factors in both sexes [20]. Their findings indicate that unlike the WC which is gender specific, a single set of values for WC/HT can be used for men and women. Nearly all overweight men and women (BMI $\geq 25)$ had WC/HT either higher or equal to 0.5 (98.5\% of men and $97.5 \%$ of women). In the same study, none of the underweight subjects had WC/HT $\geq 0.5$. Their findings indicated that utility of WC as a marker of adiposity and cardiovascular risk factor can be improved by relating it to height.

Data on the clinical utility of SFT as an anthropometric measure to estimate fat mass in adults are limited. In our study, SFT over triceps showed the strongest association with TFM and the infrascapular SFT showed the strongest association with VFM. These differences could be due to regional variation in fat accumulation. TFM and VFM showed stronger associations with all SFT measurements than WHR.

Our study has several limitations. Participants were volunteers and selection bias could have occurred during recruitment. Most of the participants (90 subjects) were doing sedentary jobs. Hence they would not be representative of people of the same age group in the community.

In conclusion, BMI was the best predictor of both TFM and VFM of subjects in this study. When compared with BMI, WC or HC were marginally less stronger in predicting fat distribution. Adjusting for height did not improve the association between fat mass and WC. In clinical settings, BMI is a more convenient and simple measurement than either WC or HC. Clinicians could use BMI as a better surrogate marker of total or visceral fat distribution in assessing and monitoring patients. The use of WHR as a surrogate of fat mass among Asians should be reconsidered. Although previous studies support our findings, we need to evaluate these associations in a larger sample to determine their reproducibility.

\section{References}

1. Pouliot MC, Despress JP, Lemieus X, Moorjani S, Bouchard C, et al. Waist circumference and abdominal sagital diameter: best simple anthropometric indices of abdominal visceral adipose tissue accumulation and related cardiovascular risk in men and women. American Journal of Cardiology 1994; 73: 460-8.

2. Bjorntorp P. Body fat distribution, insulin resistance and metabolic diseases. Nutrition 1997; 13: 795-803. 
3. Seppala-Lindroos A, Vehkavara S, Hakkineen AM, Goto T, Westerbacka J, et al. Fat accumulation in the liver is associated with defects in insulin suppression of glucose production and serum fatty acid independent of obesity in normal men. Journal of Clinical Endocrinology and Metabolism 2002; 87: 3023-8.

4. Waterlow JC. Classification and definition of protein-calorie malnutrition. British Medical Journal 1972; 5826: 566-9.

5. Shen W, Wang Z, Punyanyita M, Lei J, Sinaw A, et al. Adipose tissue quantification by imaging methods: a proposed classification. Obesity Research 2003; 11: 5-16.

6. Ford ES, Mokdad AH, Giles WH. Trends in waist circumference among US adults. Obesity Research 2003; 11: 1223-31.

7. Kamel EG, McNeill G, Van Wijk MC. Usefulness of anthropometry and DXA in predicting intra-abdominal fat in obese men and women. Obesity Research 2000; 8: 36-42.

8. Duerenberg P, Yap M, Van Staveren WA. Body mass index and percent body fat: a meta analysis among different ethnic groups. International Journal of Obesity and Metabolic Disorders 1998; 22: 164-71.

9. National Institute of Health, National Heart, Lung and Blood Institute: clinical guidelines on the identification, evaluation, and treatment of overweight and obesity in adults - the evidence report. Obesity Research 1998; 6 (Supp): 2091-5.

10. Misra A, Vikram NK. Clinical and pathophysiological consequences of abdominal adiposity and abdominal adipose tissue depots. Nutrition 2003; 19: 457-66.

11. Molarius A, Seidell JC. Selection of anthropometric indicators for classification of abdominal fatness: a critical review. International Journal of Obesity and Metabolic Disorders 1998; 22: 719-27.

12. Wagner DR, Heyward VH. Measures of body composition in blacks and whites: a comparative review. American Journal of Clinical Nutrition 2000; 71: 1392-402.
13. Thang SH, Naveed S, Mike L. ABC of obesity: assessment of obesity and its clinical implications. British Medical Journal 2006; 30: 695-8.

14. Berber A, Gomez SR, Fanghanel G, Sanchez RL. Anthropometric indices in the prediction of type 2 diabetes, high blood pressure and dyslipidemia in a Mexican population. International Journal of Obesity and Related Metabolic Disorders 2001; 25: 1794-9.

15. Hunter C, Gore CJ, Bauman A, Owen N. The relationship between body mass index and waist circumference: implications for estimates of the population prevalence of overweight. International Journal of Obesity and Related Metabolic Disorders 2000; 24: 1058-61.

16. Misra A, Misra R, Wijesuriya M, Banergee D. The metabolic syndrome in south Asians: continuing escalation and possible solutions. Indian Journal of Medical Research 2007; 125: $345-54$.

17. Wajchenberg BL. Subcutaneous and visceral adipose tissue: their relation to the metabolic syndrome. Endocrine Reviews 2000; 21: 697-738.

18. McKeigue PM, Pierpoint T, Ferrie JE, Marmot MG. Relationship of glucose intolerance and hyperinsulinaemia to body fat pattern in south Asians and Europeans. Diabetologia 1992; 35: 785-91.

19. Wang J, Thorton JC, Russell M, Burastero S, Heymsfield S, et al. Asians have lower body mass index (BMI) but higher percent body fat than do whites: comparisons of anthropometric measurements. American Journal of Clinical Nutrition 1994; 60: 23- 8

20. Hsieh SD, Muto T. Metabolic syndrome in Japanese men and women with special reference to the anthropometric criteria for the assessment of obesity: proposal to use the waist-to-height ratio. Preventive Medicine 2006; 42: 135-9. 